\title{
Histologie comparée des fosses nasales de quelques Tortues marines (Dermochelys coriacea et Chelonia mydas) et d'eaux douces (Emys orbicularis et Pseudemys scripta) (Reptilia, Dermochelyidae, Cheloniidae, Emydidae)
}

\author{
H. Saint-Girons \\ Université Pierre et Marie Curie, Laboratoire d'Evolution des Etres organisés, 105 boulevard Raspail, \\ 75006 Paris, France
}

Keywords: Testudines, nasal cavities, sensory epithelium, histology

\begin{abstract}
Comparative histological studies carried out on the nasal cavity of four species of turtles showed that the sea turtles have a more or less regressed olfactive epithelium compared to that of the Emydidae but that their vomeronasal epithelium is more developed. The location of the vomeronasal epithelium in the cavum also differs. The data suggest that the Dermochelyidae were adapted to a strict aquatic life a long time before the Cheloniidae.
\end{abstract}

\section{Résumé}

L'étude histologique des fosses nasales de quatre espèces montre que, par rapport aux Emydidae, les Tortues de mer présentent une régression plus ou moins accentuée de l'épithélium olfactif, alors que l'épithélium voméro-nasal tend au contraire à être plus développé. Sa localisation dans le cavum est également différente. L'ensemble des données suggère que les Dermochelyidae sont devenus strictement aquatiques longtemps avant les Cheloniidae.

\section{Introduction}

L'anatomie des fosses nasales des Tortues est connue depuis longtemps dans ses grandes lignes et a fait l'objet d'une excellente mise au point de Parsons (1970). Il n'en est pas de même en ce qui concerne l'histologie des épithéliums de revêtement, qui a été fort peu étudiée récemment. Toutefois, dans le cadre de recherches comparées sur les Vertébrés Tétrapodes, Graziadei \& Tucker (1970) et
Graziadei (1971) ont décrit l'ultrastructure des épithéliums sensoriels de quelques Testudinidae et Emydinae.

L'organe de Jacobson n'est pas anatomiquement individualisé chez les Tortues. Elles n'en possèdent pas moins, dans le cavum, un épithélium sensoriel de type voméro-nasal tout à fait semblable à celui de l'organe de Jacobson des Lépidosauriens et des Mammifères (Graziadei, 1971). La répartition de l'épithélium olfactif et de l'épithélium voméronasal, grossièrement connue chez quelques Tortues terrestres et d'eaux douces (Parsons, 1959), ne l'est pas chez les Tortues de mer. Or on sait que le développement respectif de ces deux types d'épithéliums varie dans une large mesure en fonction du mode de vie, notamment chez les Lépidosauriens (Gabe \& Saint Girons, 1976). C'est ce que nous avons tenté de vérifier dans le présent travail, en examinant les fosses nasales de deux Tortues de mer, dont l'une appartenant à une famille très spécialisée et encore peu connue, et de deux Tortues d'eaux douces plus au moins amphibies.

\section{Matériel et techniques}

Les têtes proviennent de très jeunes individus et ont été fixées in toto au liquide de Bouin par nos Collègues J. Lescure, C. Piaud et J. Vasse, auxquels nous tenons à exprimer notre vive reconnaissance. Le travail a porté sur quatre espèces: Dermochelys coriacea (Linnaeus, 1766) ( 2 individus), Chelonia mydas (Linnaeus, 1758) (2), Emys orbicularis (Linnaeus, 1758) (3) et Pseudemys scripta (Schoepff, 1792) (4). Après décalcification à 
l'acide trichloracétique à $5 \%$ pendant deux à cinq jours selon la taille, les têtes ont été déshydratées à l'alcool éthylique et incluses à la paraffine après passage prolongé dans l'alcool buthylique, puis débitées en coupes sériées de $10 \mu \mathrm{m}$ d'épaisseur. Des coupes transversales et longitudinales ont été faites pour toutes les espèces, ainsi que des coupes frontales pour Emys et Pseudemys. Pour chaque pièce, nous avons monté six séries de lames, en prenant une coupe sur 10 .

Ces différentes séries ont été traitées par l'acide périodique Schiff-hématoxyline-picro indigocarmin (APS-H-PI) et par l'Azan de Gabe (1968) pour l'étude topographique, par le bleu alcian à pH 2,5 et par le bleu alcian-APS pour la recherche des mucosubstances, par la tétrazoréaction de Danielli selon le mode opératoire de Pearse (1960) pour la détection des protides et par l'hémalun pour le décompte des noyaux dans les épithéliums sensoriels. Nous utiliserons ici la même classification des différents types de cellules glandulaires, basée sur les caractères histochimiques de leur produit de sécrétion, que dans un travail antérieur sur les fosses nasales des Lépidosauriens (Gabe \& Saint Girons, 1976).

Le rapport nombre de cellules réceptrices/nombre de cellules de soutien (CR/CS) a été calculé en faisant le décompte des différentes catégories de noyaux sur trois à cinq photographies prises à différents niveaux. Les valeurs absolues sont évidemment très approximatives, mais les valeurs relatives donnent probablement une assez bonne idée des capacités sensorielles des différents épithéliums. En réalité, le simple examen des préparations suffirait pour obtenir un classement tout aussi valable et les numérations facilitent surtout la comparaison avec d'autres Reptiles. Les moyennes ont été comparées par le test $t$ de Student. Dans le texte et le Tableau I, c'est l'écart type et non l'erreur standard qui figure après la moyenne.

Ce travail a été réalisé dans le cadre de l'URA 258 du CNRS.

\section{Résultats}

\section{Anatomie}

Les fosses nasales des Tortues, relativement simples, comprennent un vestibule cylindrique bien différencié et un cavum nasi proprium grossièrement ovoïde et aplati latéralement, qui se prolonge en direction ventro-caudale par un conduit nasopharyngé de longueur variable. Les narines, latérales, s'ouvrent au voisinage de l'extrémité du museau. Les fosses nasales de Emys et Pseudemys correspondent au modèle le plus classique. Le cavum, ovoïde, est simplement resserré à sa partie rostrale par une crète longitudinale sur sa face médiale. Il se prolonge, sans solution de continuité, par un conduit naso-pharyngé qui débouche sur le plafond buccal au niveau du tiers (Emys) ou des deux tiers (Pseudemys) du globe oculaire.

Par rapport aux précédentes, les fosses nasales de Chelonia présentent plusieurs caractéristiques. L'extrémité rostro-ventrale du cavum s'avance sous la partie caudale du vestibule et il existe en outre un assez large et profond diverticule dorsal. Le conduit naso-pharyngé se différencie anatomiquement audessous du cul-de-sac postérieur du cavum. Il s'ouvre sur le plafond buccal au niveau de la moitié du globe oculaire. Les fosses nasales de Dermochelys sont réduites à un tube oblique et très aplati latéralement, qui débouche largement sur le plafond buccal, juste en avant du niveau de l'oeil. Il existe cependant un diverticule dorso-médial, tout aussi large et profond que le diverticule dorsal de Chelonia. Toutes ces différences apparaissent nettement sur la Fig. 1 et on trouvera de plus amples détails, ainsi qu'une bibliographie très complête des travaux originaux, dans la mise au point de Parsons (1970).

Comme la plupart des Tortues, les Emydinae possèdent deux paires de glandes nasales externes, l'une médiale et petite qui débouche dans le cavum, l'autre dorso-latérale et plus développée qui s'ouvre à l'extrémité caudale du vestibule. Ces glandes manquent chez Chelonia et Dermochelys.

\section{Epithélium épidermique}

Le vestibule des fosses nasales des Tortues, comme celui de tous les Vertébrés Tétrapodes, est recouvert par un épithélium de type épidermique, d'une épaisseur analogue chez les quatre espèces (Tableau I). Les couches superficielles tendent toutefois à desquammer avant que ne se forme un véritable stratum corneum (Fig. 2A). Cet épithélium épidermique se retrouve sur la face ventro-médiale du conduit naso-pharyngé de Chelonia et sur la face interne des choanes de Dermochelys, alors qu'il ne dépasse pas la marge de celles-ci chez les Emydinae.

\section{Epithélium épidermoïde muqueux}

Il n'existe pas, dans les fosses nasales des Tortues, de muqueuse malpighienne proprement dite, mais 

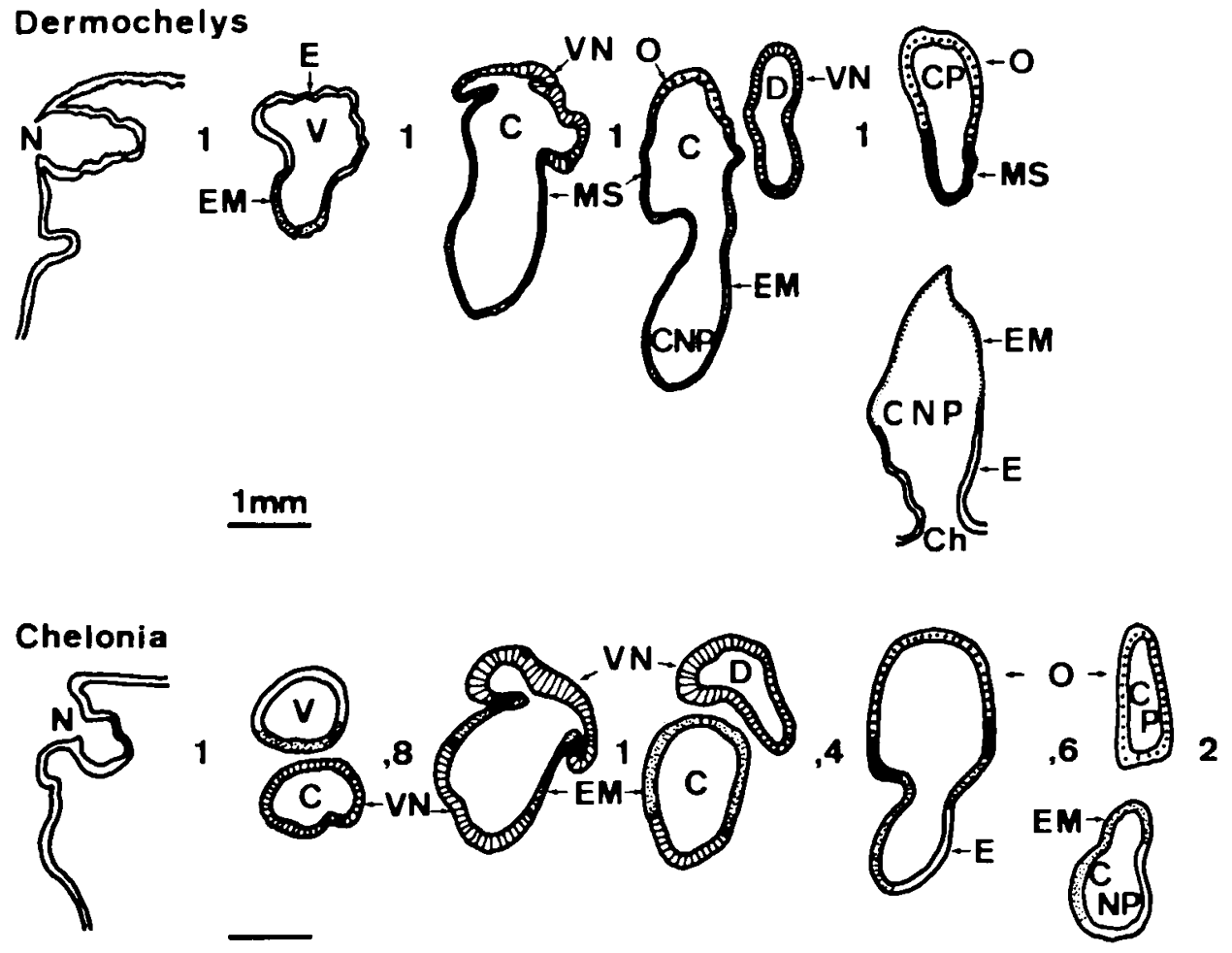

\section{Emys}

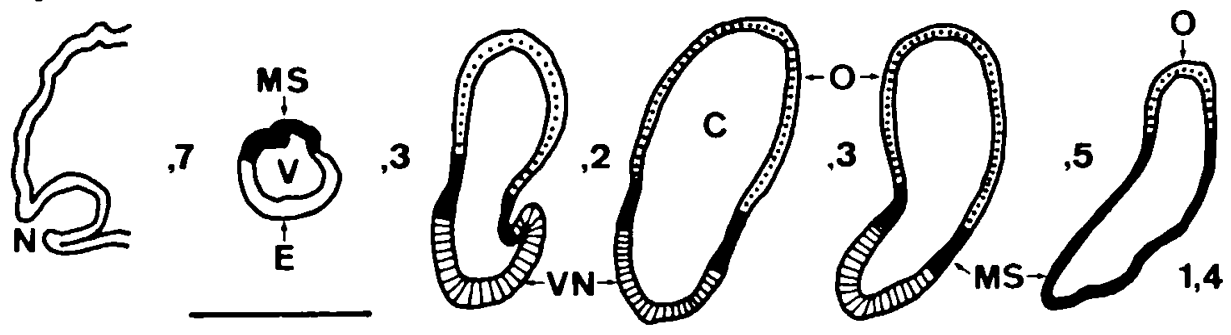

Pseudemys
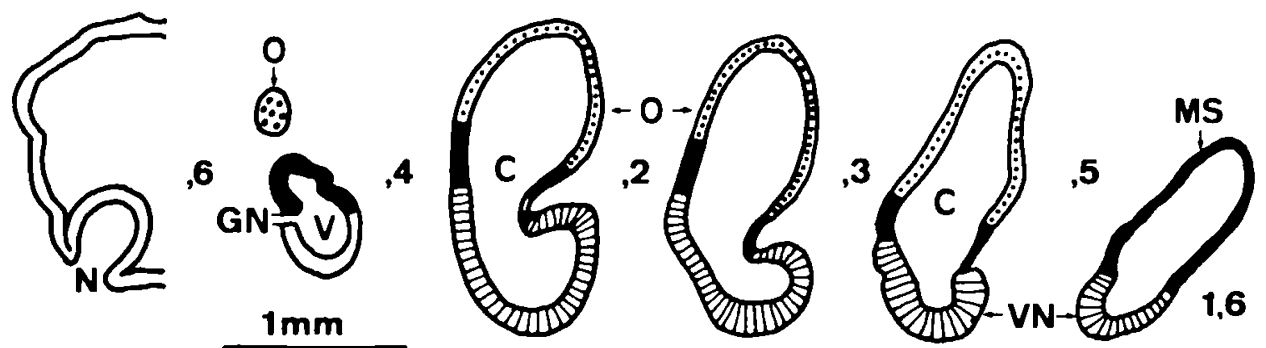

Fig. I. Coupes transversales, à des niveaux approximativement comparables, de la fosse nasale droite de Dermochelys coriacea, Chelonia mydas, Emys orbicularis et Pseudemys scripta, et répartition des différents types d'épithéliums. Les chiffres entre les coupes successives correpondent à la distance, en $\mathrm{mm}$, qui les sépare. Le dernier chiffre à droite indique la distance jusqu'à la fente antérieure de la choane. Les traits horizontaux correspondent à $1 \mathrm{~mm}$. C, cavum nasi proprium; $\mathrm{Ch}$, choane; $\mathrm{CNP}$, conduit naso-pharyngé; CP, cul-de-sac postérieur du cavum; D, diverticule dorso-médial ou dorsal du cavum; $\mathrm{GN}$, débouché de la glande nasale externe latérale; $\mathrm{N}$, narine externe; V, vestibulum naris. Les différents types d'épithéliums sont indiqués de la façon suivante: $E$, épithélium épidermique (en blanc); EM, épithélium épidermoïde muqueux (en pointillés fins); MS, épithélium muco-séreux (en noir); VN, épithélium voméro-nasal (hachuré); $\mathrm{O}$, épithélium olfactif sensu stricto (gros points). 
Tableau I. Hauteur des différents types d'épithéliums des fosses nasales des Tortues (en $\mu \mathrm{m}$ ) et rapport nombre de cellules réceptrices/nombre de cellules de soutien $(C R / C S)$ dans les épithéliums sensoriels. Pour ces derniers, moyenne, écart type et, entre parenthèses, nombre de données.

\begin{tabular}{lcccc}
\hline Epithéliums & Dermochelys & Chelonia & Emys & Pseudemys \\
\hline Epidermique & 75 à 90 & 75 à 100 & 70 à 100 & 65 à 85 \\
Epidermoïde muqueux & 60 à 80 & 75 à 130 & 75 à 90 & 75 à 90 \\
Muco-séreux & 50 à 75 & 60 à 75 & 80 à 110 & 90 à 125 \\
Voméro-nasal & 115 à 167 & 120 à 165 & 65 à 80 & 60 à 75 \\
Olfactif & 80 à 100 & 75 à 100 & & \\
$\quad$ & & $3,93 \pm 0,36(4)$ & $2,22 \pm 0,12(3)$ & $1,92 \pm 0,23(3)$ \\
$\quad$ Rapport CR/CS & $3,24 \pm 0,15(5)$ & $1,64 \pm 0,16(3)$ & $2,23 \pm 0,22(4)$ & $2,35 \pm 0,18(3)$ \\
Olfactif & 0 à 0,2 & &
\end{tabular}

deux types d'épithélium épidermoïdes muqueux (Tableaux I et II). Chez Dermochelys, deux ou parfois trois couches de petites cellules basales sont surmontées d'une couche continue de très grands mucocytes aux membranes apicales fragiles (Fig. 2B).

Chez Chelonia, l'épithélium est franchement épidermique, mais recouvert par une couche de 13 à $25 \mu \mathrm{m}$ d'épaisseur formée par une alternance de cellules muqueuses à noyaux basaux et de cellules ciliées dont les noyaux, plus ou moins allongés verticalement, sont situés nettement au-dessus des précédents (Fig. 2E). Cette couche superficielle est d'autant plus haute, en valeur relative et même absolue, que la hauteur totale de l'épithélium est plus faible (Figs. 2C, D). Dans tous les cas, le produit de sécrétion des cellules glandulaires est amorphe et correspond à une mucine acide, APS-positive, se colorant par le bleu alcian à $\mathrm{pH} 2,5$ et ne réagissant pas à la tétrazoréaction (Fig. 2F).

Cet épithélium épidermoïde muqueux recouvre toute la face ventrale du cavum de Dermochelys, depuis le débouché du vestibule, ainsi que la partie supérieure du bref conduit naso-pharyngé. Il est localisé aux faces latérales du cavum et à la moitié dorso-latérale du conduit naso-pharyngé de Chelonia, et il n'existe pratiquement pas chez les Emydinae (Fig. 1).

\section{Epithélium intermédiaire muco-séreux}

Cet épithélium, stratifié ou pseudo-stratifié, est caractérisé par la présence de cellules muco-séreuses élaborant des grains de sécrétion assez gros et bien individualisés, APS-positifs, se colorant plus ou moins intensément par le bleu alcian à $\mathrm{pH} 2,5$ et réagissant fortement à la tétrazoréaction (Tableau II, Figs. 2F, G). Ceci mis à part, la structure de cet épithélium varie beaucoup selon les régions. Il est souvent constitué par deux couches de petites cellules basales et par de grandes cellules mucoséreuses prismatiques ou ovoïdes qui alternent avec des cellules ciliées banales à noyaux plus superficiels (Fig. 2B). La hauteur de l'épithélium augmente très généralement au voisinage des zones sensorielles et son caractère pseudo-stratifié devient évident, tandis que la proportion des cellules ciliées diminue. La morphologie des cellules glandulaires dépend alors de la hauteur à laquelle sont situés leurs noyaux (Fig. 2G).

Les cellules ciliées tendent également à se raréfier lorsque la hauteur de l'épithélium diminue et, localement, celui-ci peut n'être plus constitué que par une couche de petites cellules basales et une couche régulière de grandes cellules muco-séreuses prismatiques. Enfin, dans le conduit naso-pharyngé des Emydinae, des cellules muqueuses apparaissent en nombre croissant entre les cellules mucoséreuses et les cellules ciliées. On trouve même parfois des cellules intermédiaires, c'est à dire remplies en majeure partie par un produit de sécrétion amorphe et purement muqueux, au sein duquel apparaissent quelques grains de sécrétion bien individualisés et riches en protides.

Un épithélium de type muco-séreux est toujours présent au voisinage des épithéliums sensoriels, futce sur une très étroite zone de transition, mais il tapisse également une part importante du conduit naso-pharyngé des Emydinae où il remplace, en fait, l'épithélium épidermoïde muqueux des Tortues de mer (Fig. 1). 

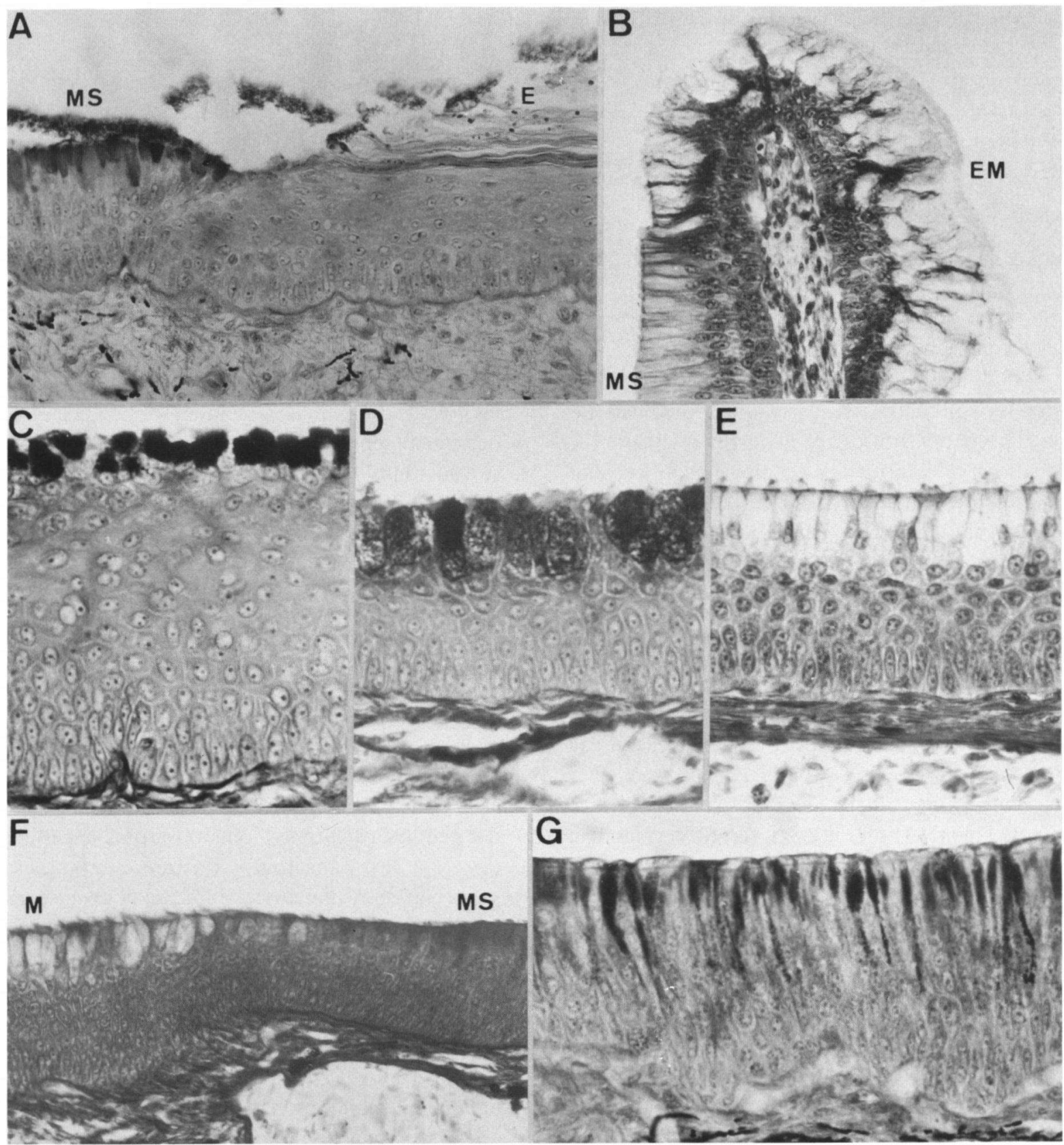

Fig. 2. Epithéliums respiratoires. Ecran vert. A, Dermochelys, APS-H-PI, $\times 250$. Passage de l'épithélium épidermique (E, à droite) à l'épithélium muco-séreux (MS, à gauche). B, Dermochelys, Azan, $\times 250$. Epithélium épidermoïde muqueux (EM), et (en bas à gauche) épithélium muco-séreux (MS). C et D, Chelonia, APS-H-PI, $\times 400$. Epithélium épidermoïde muqueux, de différentes hauteurs. E, Chelonia, Azan, $\times$ 400. Epithélium épidermoïde muqueux. F, Chelonia, tétrazoréaction, $\times 250$. Epithéliums muco-séreux (MS, à droite) et épidermoïde muqueux (M, à gauche). Noter la différence de réaction des produits de sécrétion des cellules muco-séreuses et muqueuses. G, Emys, APS-H-PI, × 400. Epithélium muco-séreux. Noter l'aspect pseudo-stratifié de l'épithélium et les grains de sécrétion APS-positifs. 
Tableau II. Caractères histochimiques des produits de sécrétion des différents types de cellules glandulaires des fosses nasales $(+=$ réaction faible; $++=$ réaction d'intensité moyenne; $+++=$ réaction forte).

\begin{tabular}{lllll}
\hline Epithéliums & Bleu alcian & APS & Tétrazoréaction & Type \\
\hline Epidermoïde muqueux & +++ & +++ & 0 & Muqueux \\
Muco-séreux & $+\mathbf{a}+++$ & $++\mathbf{a}+++$ & $++\mathbf{a}+++$ & Muco-séreux \\
Voméro-nasal & +++ & +++ & ++ & Muco-séreux \\
Olfactif & +++ & +++ & ++ & Muco-séreux \\
Glandes de Bowman & 0 & +++ & +++ & Séro-muqueux \\
\hline
\end{tabular}

\section{Epithélium voméro-nasal}

Tous les épithéliums sensoriels des fosses nasales sont pseudo-stratifiés et constitués par trois catégories cellulaires: des cellules de soutien glandulaires, des cellules réceptrices correspondant à des neurones bipolaires et des cellules basales de remplacement.

L'épithélium voméro-nasal des Tortues - comme l'épithélium sensoriel de l'organe de Jacobson des Lépidosauriens et des Mammifères - est caractérisé par l'absence de glande de Bowman et d'un film muqueux superficiel, la surface libre des cellules réceptrices étant dépourvue de cils sensoriels et garnie de microvillosités.

L'épithélium voméro-nasal de Dermochelys est haut de 115 à $170 \mu \mathrm{m}$ (Figs. 3A, B, D). Vers les deux tiers de sa hauteur, les noyaux des cellules de soutien, ovoïdes, de 4 à $6 \times 7,5$ à $9 \mu \mathrm{m}$, forment une couche régulière large de deux ou trois noyaux. La moitié inférieure de l'épithélium est occupée par les nombreux noyaux des cellules réceptrices, plus arrondis $(5 \times 6$ à $7,5 \mu \mathrm{m})$. Il existe enfin une couche discontinue de petites cellules basales dont les noyaux sont généralement plus petits et plus irréguliers que les précédents. Les axones se distinguent mal en microscopie optique. Il n'en est pas de même pour la partie supra-nucléaire des cellules de soutien, large de 3 à $4 \mu \mathrm{m}$ et plus ou moins chargée de grains de sécrétion bien individualisés, APS-positifs, se colorant par le bleu alcian à pH 2,5 et riches en protides, très semblables à ceux des cellules mucoséreuses de l'épithélium intermédiaire décrit précédemment. Le rapport nombre de cellules réceptrices/nombre de cellules de soutien (CR/CS) varie de 3,05 à $3,38(\bar{x}=3,24 \pm 0,15)$.
L'épithélium voméro-nasal de Chelonia est très semblable à celui de Dermochelys, à ceci près que les noyaux des cellules de soutien sont un peu moins nettement séparés de ceux des cellules réceptrices, surtout dans la partie rostrale des fosses nasales (Fig. 3C). Le rapport CR/CS est compris entre 3,51 et $4,31(\bar{x}=3,93 \pm 0,36)$, c'est à dire que les cellules réceptrices sont proportionnellement un peu plus nombreuses que chez Dermochelys, la différence étant très significative $(p<0,01)$.

L'épithélium voméro-nasal des Emydinae est un peu moins haut que les précédents (Tableau I). Les noyaux des cellules de soutien, plus allongés ( 3 à 4 $\times 9$ à $11 \mu \mathrm{m}$ ) et plus riches en chromatine, forment une couche assez régulière entre le tiers supérieur et le milieu de l'épithélium (Fig. 3E). Les noyaux des cellules réceptrices, plus ou moins sphériques $(6 \times 6$ à $7 \times 7$, parfois $5 \times 8 \mu \mathrm{m})$ et assez clairs, s'étagent immédiatement au-dessous et jusqu'au voisinage de la membrane basale, mais un certain nombre d'entre eux sont mélangés aux noyaux des cellules de soutien. Le rapport $C R / C S$ varie de 1,69 à $2,35(\bar{x}=2,22 \pm 0,12$ chez Emys et 1,92 $\pm 0,23$ chez Pseudemys, différence non significative). La proportion des cellules réceptrices est donc nettement plus faible que chez les Tortues de mer, la différence étant très significative dans tous les cas $(p<0,01)$. Les grains de sécrétion des cellules de soutien, plus abondants chez Pseudemys, ne diffèrent pas de ceux des Tortues de mer. Des capillaires pénêtrent toujours dans l'épithélium voméro-nasal et d'autant plus profondément que celui-ci est plus haut. Toutefois, l'épithélium n'est pas découpé en petites sous-unités régulières, comme c'est le cas pour l'épithélium sensoriel de l'organe de Jacobson des Squamates. 


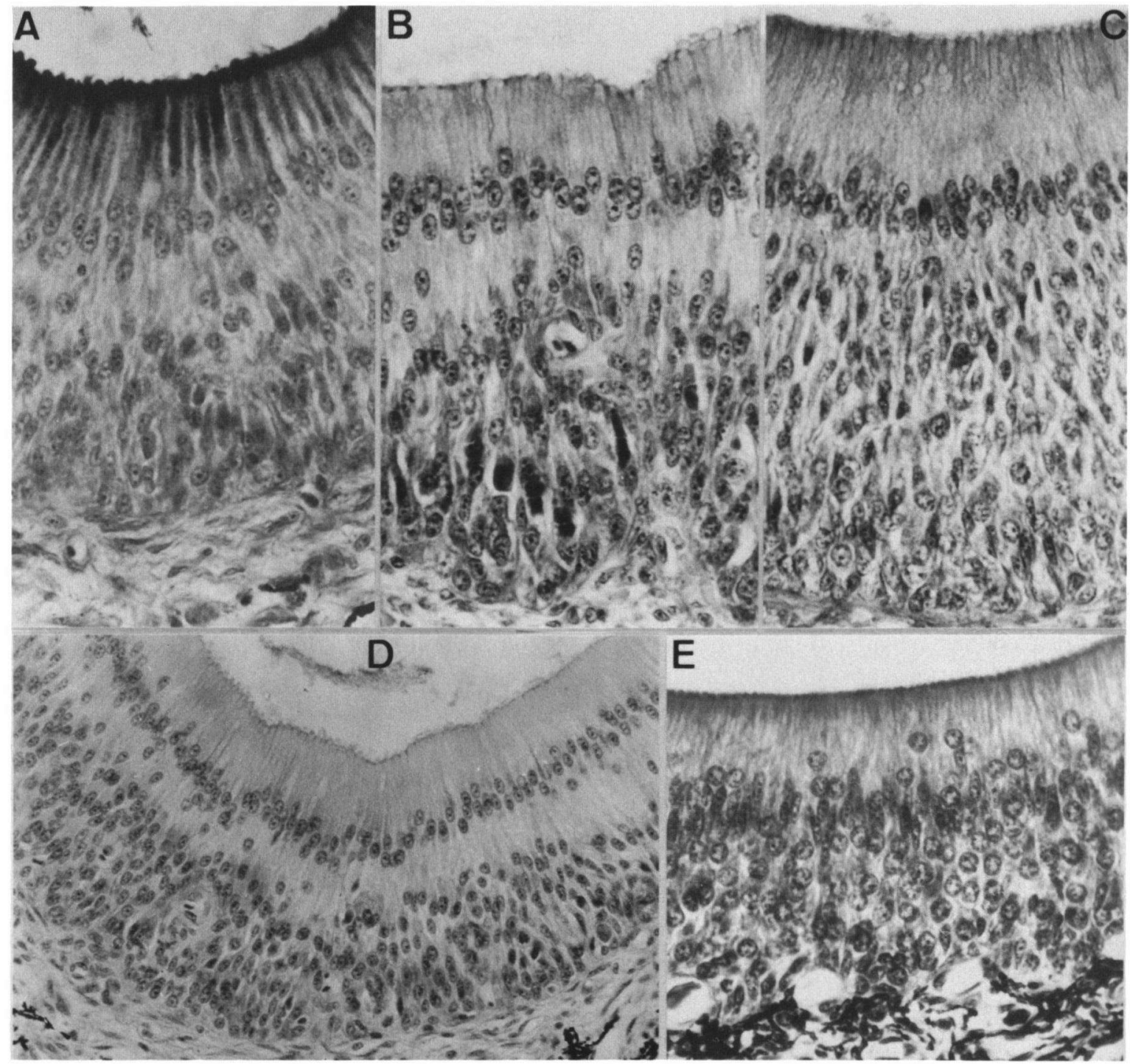

Fig. 3. Epithélium voméro-nasal. Ecran vert. A, Dermochelys, APS-H-PI, $\times 400$. B, Dermochelys, Azan, $\times 400$. C, Chelonia, Azan, $\times 400$. D, Dermochelys, hémalun, $\times 250 . \mathrm{E}$, Emys, Azan, $\times 400$.

L'épithélium voméro-nasal tapisse la face antéro-dorsale du cavum et tout le diverticule médio-dorsal de Dermochelys. Outre cette zone, il recouvre également chez Chelonia le cul-de-sac rostral et la face rostro-ventrale du cavum (Fig. 1). L'épithélium voméro-nasal des Emydinae est localisé à la face ventrale du cavum et, chez Pseudemys, il dépasse caudalement le niveau de l'épithélium olfactif. Par rapport à l'ensemble du cavum, l'étendue relative de l'épithélium voméro-nasal est mani- festement beaucoup plus grande chez Chelonia que chez Dermochelys et un peu plus grande chez Pseudemys que chez Emys. La comparaison entre les Tortues de mer et les Emydinae est plus difficile, en raison des différences de structure du cavum. Il est clair que l'étendue relative de l'épithélium voméro-nasal est minimal chez Dermochelys, mais de ce point de vue Chelonia peut se situer soit au même niveau que les Emydinae, soit un peu audessus. 


\section{Epithélium olfactif}

La structure générale de l'épithélium olfactif sensu stricto est analogue à celle de l'épithélium voméronasal, mais il en diffère par l'existence de nombreuses glandes de Bowman et de très longs cils sensoriels des cellules réceptrices, qui s'étalent au voisinage de la surface d'une mince couche de mucus. L'épithélium olfactif de Emys et Pseudemys, de structure classique, est haut de 65 à $80 \mu \mathrm{m}$ (Tableau I). Les noyaux des cellules de soutien, ovoïdes, de 4 à $5 \times 8$ à $10 \mu \mathrm{m}$, sont situés vers le tiers ou le quart supérieur de sa hauteur (Figs. 4B, D). Les noyaux des cellules réceptrices, plus arrondis (5 à $6 \mu \mathrm{m}$ de diamètre), s'étendent immédiatement au-dessous et jusqu'à la couche discontinue de petites cellules basales. Le produit de sécrétion des cellules de soutien, assez abondant dans la région supra-nucléaire, est en tous points identique à celui de l'épithélium voméro-nasal. Le rapport $\mathrm{CR} / \mathrm{CS}$ varie de 1,91 à $2,52(\bar{x}=2,23 \pm 0,22 \mathrm{chez}$ Emys, 2,35 $\pm 0,18$ chez Pseudemys).

L'épithélium olfactif de Chelonia, haut de 75 à $100 \mu \mathrm{m}$, ressemble assez à celui des Emydinae, mais la proportion des cellules réceptrices y est nettement moindre. En effet, le rapport $\mathrm{CR} / \mathrm{CS}$ est compris entre 1,47 et $1,78(\bar{x}=1,64 \pm 0,16)$, la différence avec Emys ou Pseudemys étant significative ( $p<$ $0,02)$. Par ailleurs, les noyaux des cellules de soutien, plus arrondis, ressemblent davantage à ceux des cellules réceptrices. Bien que tout ainsi haut que les précédents, l'épithélium olfactif de Dermochelys ne mérite peut-être pas ce nom, car les cellules réceptrices y sont très rares, sinon même absentes (Figs. 4A, C). Il existe, dans la moitié inférieure de l'épithélium, entre la bande assez large de noyaux de cellules de soutien et la couche discontinue des petites cellules basales, quelques noyaux dont nous ne pouvons dire s'ils correspondent à des cellules réceptrices, ou bien s'ils appartiennent à des cellules de soutien et sont en cours d'ascension. De toute façon, si elles existent, les cellules réceptrices sont manifestement rares, avec un rapport CR/CS compris entre 0 et 0,2 . Les cils sensoriels n'apparaissent pas nettement à la surface de l'épithélium et, s'il y en a, leur nombre est trop faible pour maintenir en place la fine couche de mucus superficielle qui est caractéristique de l'épithélium olfactif proprement dit. L'abondance et la nature des grains de sécrétion des cellules de soutien des Tortues de mer ne diffèrent pas de ceux de Emydinae.

Les glandes de Bowman, nombreuses, sont en majeure partie extra-épithéliales (Fig. 4B). Leurs grains de sécrétion, très abondants, sont assez gros et bien individualisés, riches en protides et APSpositifs, mais ils ne se colorent pas par le bleu alcian à $\mathrm{pH} 2,5$. Ils sont donc séro-muqueux, comme d'ailleurs chez tous les Vertébrés Tétrapodes. Les cellules du collet sont, comme d'ordinaire aussi, muco-séreuses. De structure classique chez les Emydinae et Chelonia, les glandes de Bowman de Dermochelys ont une lumière anormalement large, jusque et y compris souvent dans leur trajet intraépithélial (Figs. 4A, C). La couche de mucus, haute de 4 à $6 \mu \mathrm{m}$, qui recouvre l'épithélium olfactif des Emydinae et de Chelonia est APS-positive, se colore par le bleu alcian à $\mathrm{pH} 2,5$ et réagit fortement à la tétrazoréaction. Elle est donc de type mucoséreux et, selon toute vraisemblance, constituée par un mélange des produits de sécrétion des cellules de soutien et des glandes de Bowman.

L'épithélium olfactif tapisse régulièrement la moitié dorsale du cavum des Emydinae, son expansion latérale étant un peu plus grande chez Emys que chez Pseudemys. Il est, en revanche, localisé à la partie dorso-caudale du cavum des Tortues de mer. Sa superficie relative est minimale chez Dermochelys, légèrement supérieure chez Chelonia, très supérieure chez Pseudemys et probablement encore un peu plus chez Emys.

\section{Discussion}

En ce qui concerne l'anatomie des fosses nasales des Tortues, nous renvoyons à la revue détaillée de Parsons (1970). Rappelons simplement que le vestibulum naris, constitué par un simple tube cylindrique, est généralement court, sauf chez quelques Tortues d'eaux douces au museau allongé, comme Chelus et les Trionychidae. Le cavum nasi proprium des Tortues de mer et surtout de Dermochelys est proportionnellement moins volumineux que celui des autres représentants de l'ordre, mais il est pourvu d'un profond diverticule dorsal, ou dorso-médial. Le 


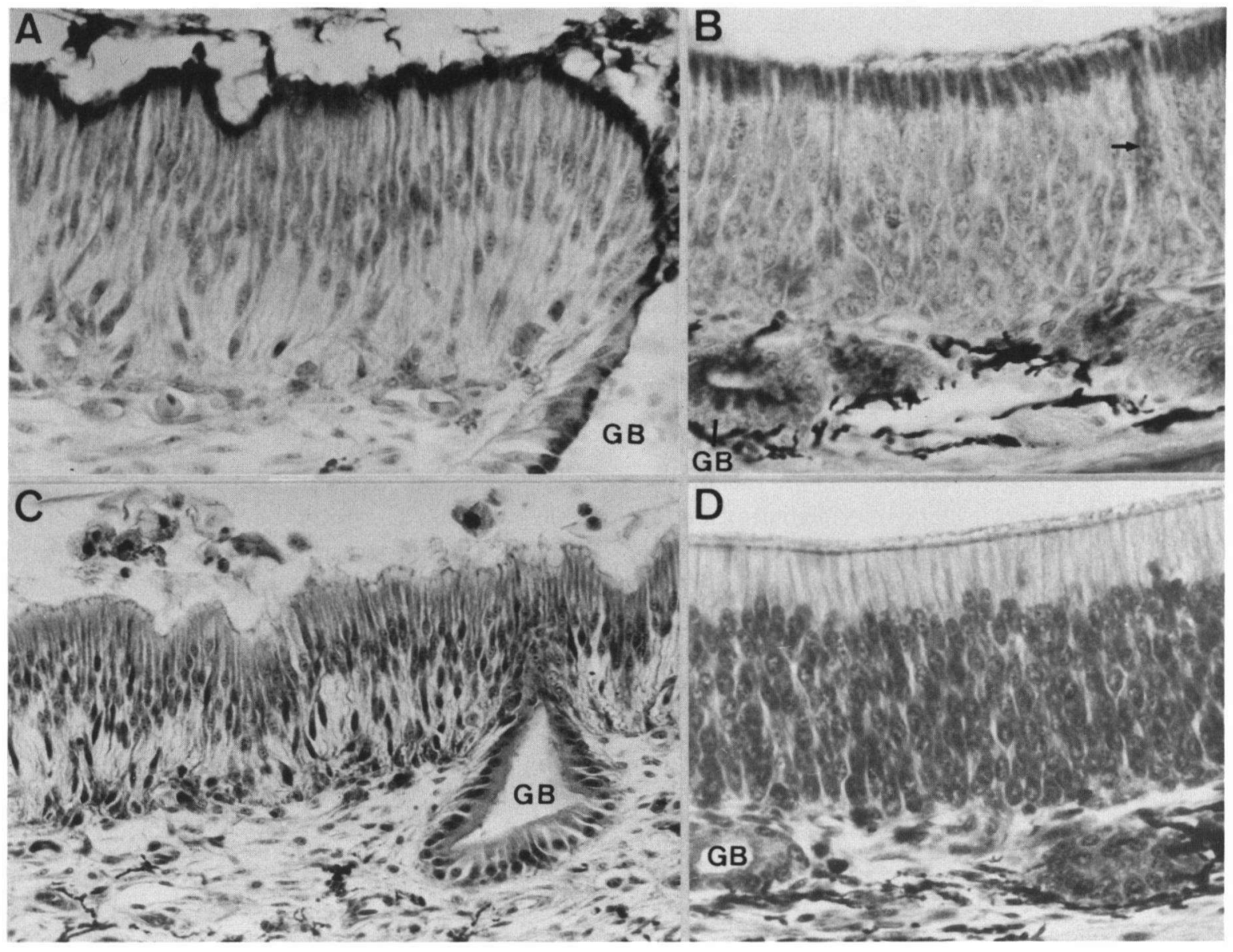

Fig. 4. Epithélium olfactif et glandes de Bowman (GB). Ecran vert. A, Dermochelys, APS-H-PI, $\times 400$. A droite, le débouché d'une glande de Bowman. B, Emys, APS-H-PI, $\times 400$. La flèche indique le conduit excréteur, intra-épithelial, d'une glande de Bowman. C, Dermochelys, Azan, $\times 250 . \mathrm{D}$, Emys, Azan $\times 400$. Les cils sensoriels des cellules réceptrices apparaissent nettement à la surface de l'épithélium.

récessus rostro-ventral qui s'avance sous la partie postérieure du vestibule paraît en revanche propre aux Cheloniidae. Le conduit naso-pharyngé est plus ou moins long et, tout en se rétrécissant progressivement, il prolonge habituellement sans solution de continuité le cavum vers l'arrière. Il est un peu mieux différencié chez les Testudinidae et les Cheloniidae et, au contraire, presque absent chez Dermochelys.

Les Tortues possèdent généralement deux paires de glandes nasales externes, l'une médiale, l'autre latérale. Ces glandes manquent chez les Tortues de mer, elles paraissent peu développées chez les Trionychidae (Parsons, 1971) et plus volumineuses chez les Testudinidae que les Emydidae. La régression allant jusqu'à la disparition des glandes na- sales externes, comme d'ailleurs de beaucoup d'autres glandes céphaliques exocrines, est un phénomène déjà noté chez les Reptiles aquatiques plus ou moins spécialisés (Saint Girons, 1988; 1989).

La répartition des différents types d'épithéliums est beaucoup moins bien connue, surtout dans le cavum. Comme chez tous les Reptiles, l'épithélium épidermique définit le vestibule plus sûrement que la simple anatomie, de même que le conduit nasopharyngé est par définition recouvert d'un épithélium de type respiratoire. On admet généralement que le cavum de la plupart des Tortues est tapissé sur sa partie dorsale par un épithélium olfactif pourvu de glandes de Bowman et sur sa partie ven- 
trale par un épithélium voméro-nasal, ces deux types d'épithéliums étant séparés par des bandes plus ou moins larges d'épithélium "respiratoire", c'est à dire non sensoriel (Parsons, 1959; 1970). C'est, effectivement, ce que nous avons constaté chez Emys et Pseudemys.

Mais, la situation est toute différente chez les Tortues de mer, car l'épithélium olfactif est localisé à la partie dorso-caudale du cavum, tandis que sa partie dorso-rostrale et le profond diverticule qui s'y trouve sont tapissés par un épithélium voméronasal. Ce dernier s'étend toutefois, chez Chelonia, sur toute la zone rostro-ventrale du cavum. Cette espèce, et probablement tous les Cheloniidae, pourrait donc représenter un stade intermédiaire dans la migration de l'épithélium voméro-nasal de la face ventrale à la face dorso-rostrale du cavum chez les Tortues de mer, migration permise, ou provoquée, par la régression de l'épithélium olfactif. En effet, malgré l'absence de mesure précise, il apparaît nettement que l'étendue relative de l'épithélium olfactif est beaucoup plus faible chez les Tortues de mer que chez les Emydinae. Il n'en est pas de même pour l'épithélium : voméro-nasal dont l'étendue relative, certes modérée chez Dermochelys, paraît chez Chelonia aussi grande, sinon plus, que chez les Emydinae.

D'un point de vue histologique, l'épithélium épidermique du vestibule semble analogue chez toutes les Tortues et d'ailleurs tous les Reptiles. L'épithélium respiratoire qui recouvre le conduit naso-pharyngé et une partie plus ou moins grande du cavum n'a pas, à notre connaissance, fait l'objet d'étude permettant de comparer notre matériel à d'autres Tortues. Il convient cependant de noter les différences importantes qui existent entre les Emydinae, pourvus d'un seul type d'épithélium respiratoire, muco-séreux, et les Tortues de mer chez lesquelles cet épithélium muco-séreux est faiblement représenté, les zones "respiratoires" étant en majorité recouvertes par un épithélium épidermoïde muqueux ou, à la partie postérieure du conduit naso-pharyngé, par un épithélium épidermique. Les différences de structure des épithéliums épidermoïdes muqueux entre Dermochelys et Chelonia rendent difficile une appréciation fonctionnelle et, de ce point de vue, il serait intéressant d'étudier des Tortues d'eaux douces plus adaptées à la vie aquatique que les Emydinae.

En ce qui concerne la structure générale des épithéliums sensoriels des Tortues, notamment les différences qui existent entre l'épithélium olfactif et l'épithélium voméro-nasal, nos données confirment les observations faites par Graziadei et Tucker (1970) et par Graziadei (1971) sur quelques Testudinidae et Emydinae. Si l'on en juge par les figures de ces auteurs, l'épithélium olfactif de la Tortue terrestre Gopherus polyphemus (Daudin, 1803) est très comparable à celui de Emys et de Pseudemys, la proportion des cellules réceptrices étant peut-être légèrement supérieure. Bien que moins développé que celui des Emydinae, l'épithélium olfactif de Chelonia semble normal et fonctionnel. En revanche, et c'est le phénomène le plus intéressant que nous ayons mis en évidence, l'épithélium olfactif de Dermochelys est manifestement régressé et presque certainement non fonctionnel.

L'épithélium voméro-nasal, lui, paraît bien développé chez toutes les Tortues examinées à cet égard, c'est à dire, outre le présent matériel, quelques Emydinae et Gopherus polyphemus (cf. Parsons, 1970; Graziadei \& Tucker, 1970). Les cellules réceptrices sont toujours plus nombreuses que les cellules de soutien. Toutefois, le rapport CR/CS varie de façon significative entre les espèces, la proportion des cellules réceptrices décroissant dans l'ordre Chelonia, Dermochelys, Emys et Pseudemys. D'après les données morphologiques, il est certain que l'épithélium voméro-nasal joue un rôle sensoriel important chez les Tortues. A peu près aussi développé que l'épithélium olfactif chez les Emydinae, il l'est davantage chez les Tortues de mer et surtout Dermochelys où l'épithélium olfactif est profondément régressé.

Il est toujours difficile, lors de la comparaison de structures morphologiques, de savoir quelle est, dans les différences observées, la part qui revient au mode de vie, donc à des adaptations fonctionnelles pouvant relever de phénomènes de convergence, de celle qui est due à la position systématique. En ce qui concerne les fosses nasales, Chelonia se rapproche de Dermochelys par la présence d'un profond diverticule dorsal dans le cavum, le développement important de l'épithélium voméro-nasal, la 
forte réduction de l'épithélium respiratoire mucoséreux remplacé par un épithélium épidermoïde muqueux et la disparition des glandes nasales externes. Le volume relatif du cavum et le développement de l'épithélium olfactif placent Chelonia en position intermédiaire entre Dermochelys et les Emydinae, tandis que l'existence d'un véritable conduit naso-pharyngé rapproche au contraire Chelonia de ces derniers.

La présence d'un récessus rostro-ventral du cavum est, en revanche, un caractère propre aux Cheloniidae, puisqu'il a également été noté chez Eretmochelys (in Parsons, 1970). Tous les auteurs sont d'accord pour admettre que Dermochelys, unique représentant de la famille, est la plus spécialisée des Tortues de mer. On peut donc considérer que la simplification des fosses nasales et l'extrême réduction de l'épithélium olfactif sont dues au mode de vie, d'autant que ces tendances se marquent déjà chez Chelonia. On les retrouve d'ailleurs chez les Serpents aquatiques très spécialisés, tels que les Acrochordidae et les Hydrophiinae, mais non chez les Reptiles plus ou moins amphibies, qu'ils soient marins comme les Laticaudinae, ou d'eaux douces comme de nombreux Colubridae ou les Crocodiles (Gabe \& Saint Girons, 1976; Saint Girons, 1976).

L'évolution de l'épithélium voméro-nasal est moins claire. On sait que l'organe de Jacobson des Serpents aquatiques les plus spécialisés échappe à la régression qui frappe l'épithélium olfactif, mais il n'est pas plus développé que celui des Serpents amphibies ou terrestres. Parmi les Tortues, il semble au premier abord que l'épithélium voméro-nasal soit plus développé chez les formes aquatiques très spécialisées. Mair, curieusement, c'est chez Chelonia, et non Dermochelys, qu'il l'est le plus. Deux hypothèses, entre autres, peuvent expliquer cette situation a priori inattendue. L'une est que, après être passée par un stade de type Chelonia, Dermochelys, en se spécialisant de plus en plus, tende à devenir anosmate comme, par exemple, les Cétacés. L'autre est que le moindre développement de l'épithélium voméro-nasal de cette espèce est dû à un régime presque exclusivement composé de Méduses, proies dont la capture et l'ingestion ne nécessitent peut-être pas des sens chimiques performants et que Dermochelys confond d'ailleurs, malheureusement pour elle, avec des sacs de plastique.

Si l'on en juge par l'exemple des Serpents, Reptiles aux modes de vie très variés, la simplification des fosses nasales et en particulier la régression de l'épithélium olfactif, sont limitées aux groupes aquatiques les plus spécialisés et se manifestent sans doute très lentement. Dans cette optique, on peut penser que les Dermochelyidae, qu'ils aient ou non la même origine que les Cheloniidae, sont devenus strictement marins bien avant eux.

\section{Bibliographie}

Gabe, M., 1968. Techniques histologiques: 1-1113 (Masson, Paris).

Gabe, M. \& H. Saint Girons, 1976. Contribution à la morphologie comparée des fosses nasales et de leurs annexes chez les Lépidosauriens. Mém. Mus. natn. Hist. nat., (A) 98: $1-87$, figs. 1-49, pls. I-X.

Graziadei, P.P.C., 1971. The olfactory mucosa of vertebrates. In: L.M. Beidler, ed., Handbook of sensory physiology, 4: 27-58 (Springer Verlag, Berlin).

Graziadei, P.P.C. \& D. Tucker, 1970. Vomeronasal receptors in turtles. Z. Zellforsch., 105: 498-514.

Parsons, T.S., 1959. Studies on the comparative embryology of the reptilian nose. Bull. Mus. comp. Zool. Harvard, 120: 101-277.

Parsons, T.S., 1970. The nose. In: C. Gans \& T.S. Parsons, eds., Biology of the Reptilia, 2: 99-191 (Academic Press, London).

Parsons, T.S., 1971. Nasal anatomy of trionychid turtles. J. Morphol., 135: 323-333.

Pearse, A.G.E., 1960. Histochemistry: 1-998 (Churchill, London).

Saint Girons, H., 1976. Données histologiques sur les fosses nasales et leurs annexes chez Crocodylus niloticus Laurenti et Caiman crocodilus (Linnaeus) (Reptilia, Crocodylidae). Zoomorphologie, 84: 301-318.

Saint Girons, H., 1988. Les glandes céphaliques exocrines des Reptiles. I. Données anatomiques et histologiques. Annls. Sci. nat. Zool., (13)9: 221-255.

Saint Girons, H., 1989. Les glandes céphaliques exocrines des Reptiles. II. Considérations fonctionnelles et évolutives. Annls. Sci. nat. Zool., (13)10: 1-17.

Reçu le 26 mars 1990 